\author{
SERIES "ADVANCES IN PATHOBIOLOGY, DIAGNOSIS, AND TREATMENT OF \\ PULMONARY HYPERTENSION" \\ Edited by A.T. Dinh-Xuan, M. Humbert and R. Naeije \\ Number 1 in this Series
}

\title{
Genetics of pulmonary hypertension: from bench to bedside
}

\author{
M. Humbert*, R.C. Trembath ${ }^{\#}$
}

\begin{abstract}
Genetics of pulmonary hypertension: from bench to bedside. M. Humbert, R.C. Trembath. (C) ERS Journals Ltd 2002.

ABSTRACT: Primary pulmonary hypertension has been described as either sporadic or clustered in families. Familial primary pulmonary hypertension segregates as an autosomal dominant trait with markedly reduced disease gene penetrance. Defects within bone morphogenetic protein receptor type II gene, coding for a receptor member of the transforming growth factor- $\beta$ family, underlie familial primary pulmonary hypertension. Several lines of evidence point to the potential requirement of additional factors, either environmental or genetic, in the pathogenesis of the disease. In addition, a proportion of so-called sporadic primary pulmonary hypertension turns out to have an inherited basis, as demonstrated by germline bone morphogenetic protein receptor type II gene mutations. Analysis of cases in association with hereditary haemorrhagic telangiectasia led to the demonstration that pulmonary arterial hypertension can involve activin-receptor-like kinase 1 mutations, a type I transforming growth factor- $\beta$ receptor. These findings emphasise the critical role of the transforming growth factor- $\beta$ signalling pathway in pulmonary arterial hypertension. While this achievement has generated extreme interest, the pathobiology of severe pulmonary arterial hypertension remains unclear and genomic approaches to pulmonary hypertension research may identify additional molecular determinants for this disorder. Finally, there is an urgent need to develop relevant guidelines for genetic counselling to assist patients, their relatives and pulmonary vascular specialists to utilise these recent observations. Eur Respir J 2002; 20: 741-749.
\end{abstract}

\begin{abstract}
*UPRES 2705, Service de Pneumologie et Réanimation Respiratoire, Centre des Maladies Vasculaires Pulmonaires, Hôpital Antoine-Béclère, Université Paris-Sud, Clamart. " Division of Medical Genetics, Depts of Medicine and Genetics, University of Leicester, Leicester, UK
\end{abstract}

Correspondence: M. Humbert, Service de Pneumologie, Centre des Maladies Vasculaires Pulmonaires, Hôpital Antoine Béclère, 157 rue de la Porte de Trivaux, 92140 Clamart, France. Fax: 33146303824

E-mail: humbert@ipsc.u-psud.fr

Keywords: Activin-receptor-like kinase 1 , bone morphogenetic protein receptor type II gene, fenfluramine derivatives, hereditary haemorrhagic telangiectasia, primary pulmonary hypertension, pulmonary arterial hypertension

Received: April 22002

Accepted after revision: April 192002

M. Humbert and R.C. Trembath were supported in part by Université ParisSud, University of Leicester, MRC (UK), BHF, INSERM and AFM.
The normal adult pulmonary circulation is a highly distensible, low resistance, low pressure circuit with little or no resting vascular tone. The thin walls of pulmonary vessels, the lack of well-developed arterioles, and the crescentic shape of the thin-walled right ventricle reflect the low ambient haemodynamic stress. Pulmonary arterial hypertension (PAH) refers to a spectrum of diseases in which the pulmonary artery pressure is persistently elevated (mean pulmonary artery pressure $>25 \mathrm{mmHg}$ ), in the absence of a raised pulmonary artery wedge pressure $(<12 \mathrm{mmHg})$, excluding a post capillary process (mostly represented by left heart disease) $[1,2]$. This severe disorder leads to progressive right heart hypertrophy, dilatation and failure. PAH typically results from chronic obstruction of small pulmonary arteries due at least in part to

For editorial comments see page 509. endothelial and vascular smooth muscle cell dysfunction and proliferation $[2,3]$.

A revised diagnostic classification of $\mathrm{PAH}$ has been recently proposed $[2,4]$. This classification reflects recent advances in the understanding of pulmonary vascular diseases, and recognises the pathophysiological and therapeutic similarities between "unexplained" pulmonary hypertension (primary or idiopathic pulmonary hypertension) and PAH coexisting with other disorders such as collagen vascular diseases (scleroderma spectrum, systemic lupus erythematosus, mixed connective tissue disease), human immunodeficiency (HIV) infection, portal hypertension, congenital systemic to pulmonary shunts, and anorexigen exposure (table 1). In addition, this classification identifies conditions resulting from other mechanisms for which specific therapies exist, including chronic thromboembolic disease (obstructive pulmonary hypertension which can be cured by thromboendarteriectomy), chronic 
Table 1. - The Evian diagnostic classification of pulmonary hypertension

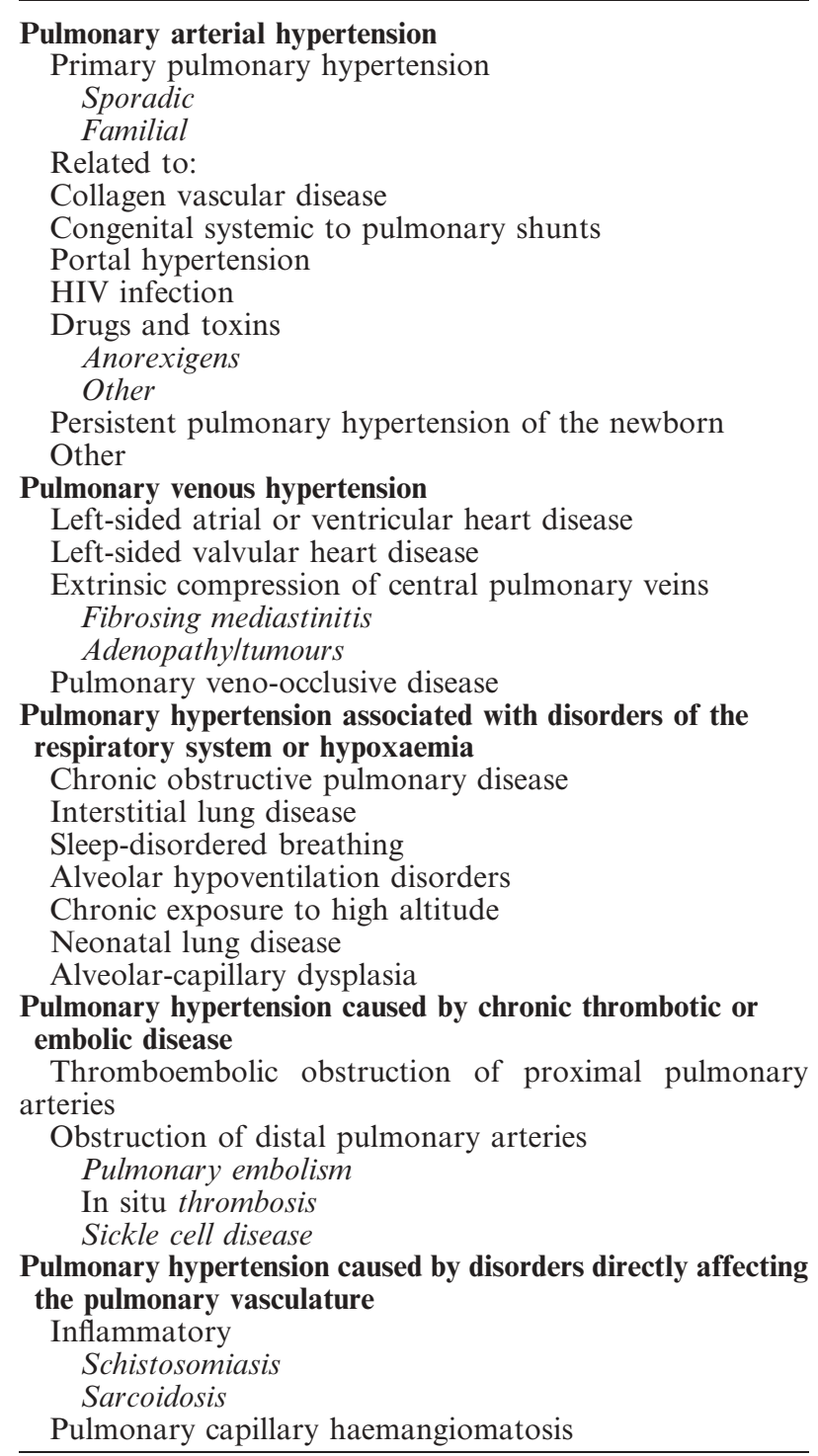

HIV: human immunodeficiency virus.

hypoxic lung disease (vasoconstrictive pulmonary hypertension which may benefit from long-term oxygen therapy) and left-sided cardiac disease (passive postcapillary venous pulmonary hypertension, which can deteriorate with prostanoids with a risk of pulmonary oedema).

Primary pulmonary hypertension $(\mathrm{PPH})$ is an uncommon disorder, with an estimated yearly incidence of 2 per million people [5]. $\mathrm{PPH}$ is twice as common in females than males and symptoms develop typically in the third and fourth decades of life, although the disease may occur at any age [6]. The mainstay of treatment is anticoagulants and pulmonary vasodilators including calcium channel antagonists, prostaglandins (epoprostenol and other prostacyclin analogues), and endothelin receptor antagonists. When medical therapy is ineffective or unavailable, lung or heart-lung transplantation is currently the only alternative therapy. Until recently, the cellular and molecular basis of this devastating disease has remained elusive.

\section{Familial primary pulmonary hypertension}

PPH can be either sporadic or clustered in families. The first detailed description of familial PPH by DresDale et al. [7] in 1954 included a description of related subjects with severe pulmonary vascular disease of unknown aetiology. Later, it became apparent that this familial condition was less rare than initially believed, in part because of patient and physician unawareness of the familial occurrence of the disease, but also because of the markedly reduced penetrance of the genetic defects [2]. A pioneering report by LoYD et al. [8] in 1984, clearly supported the concept that a proportion of cases of so-called sporadic PPH were in fact mislabelled as nonfamilial when family histories were incomplete, suggesting that the incidence of familial PPH is higher than previously reported. Because of the lack of data, the true incidence of familial PPH is unknown. The best estimate has been provided by the National Institute of Health Registry for PPH reported by RICH et al. [9] in 1987. This prospective analysis of $187 \mathrm{PPH}$ patients from 32 North American medical centres identified 12 cases with familial history affecting a first-blood relative. Consequently, it has been widely accepted that $\geqslant 6 \%$ of individuals diagnosed with $\mathrm{PPH}$ have a family history of the disorder, and thereby identifying first-degree relatives as being at additional risk of developing the disorder. Familial $\mathrm{PPH}$ segregates as an autosomal dominant trait but with markedly reduced penetrance [2, 8]. Recognition of an inherited form of this disorder enabled chromosomal localisation using linkage analysis, the locus designated PPH1 on chromosome 2q31-33 [10]. The completion of the first draft of the Human Genome Project and the contribution of additional patients and families contributed to the discovery of the PPH1 gene in the year 2000, using positional cloning. The bone morphogenetic protein receptor type II (BMPR-II) gene (BMPR2), coding for a receptor member of the transforming growth factor (TGF)- $\beta$ family, has been identified as causative of familial PPH [11, 12]. BMPR-II is a ubiquitously expressed receptor for a family of secreted growth factors named bone morphogenetic proteins (BMPs) [13] (fig. 1). These proteins are part of the TGF- $\beta$ superfamily, which include the three mammalian TGF- $\beta$ isoforms, the activins and inhibins, together with $>30$ members of the BMP subfamily [13]. BMPs were originally identified as proteins regulating growth and differentiation of bone and cartilage but more recent studies have revealed that these multifunctional cytokines regulate growth, differentiation and apoptosis of various cell types. Members of the TGF- $\beta$ family signal by binding to type I and II cell surface receptors and activating serine/threonine kinases by formation of heteromeric complexes [14, 15].

By direct sequence analysis of the protein-encoding portion of the BMPR2 gene in PPH patients, germline heterozygous missense, nonsense splice-site and 


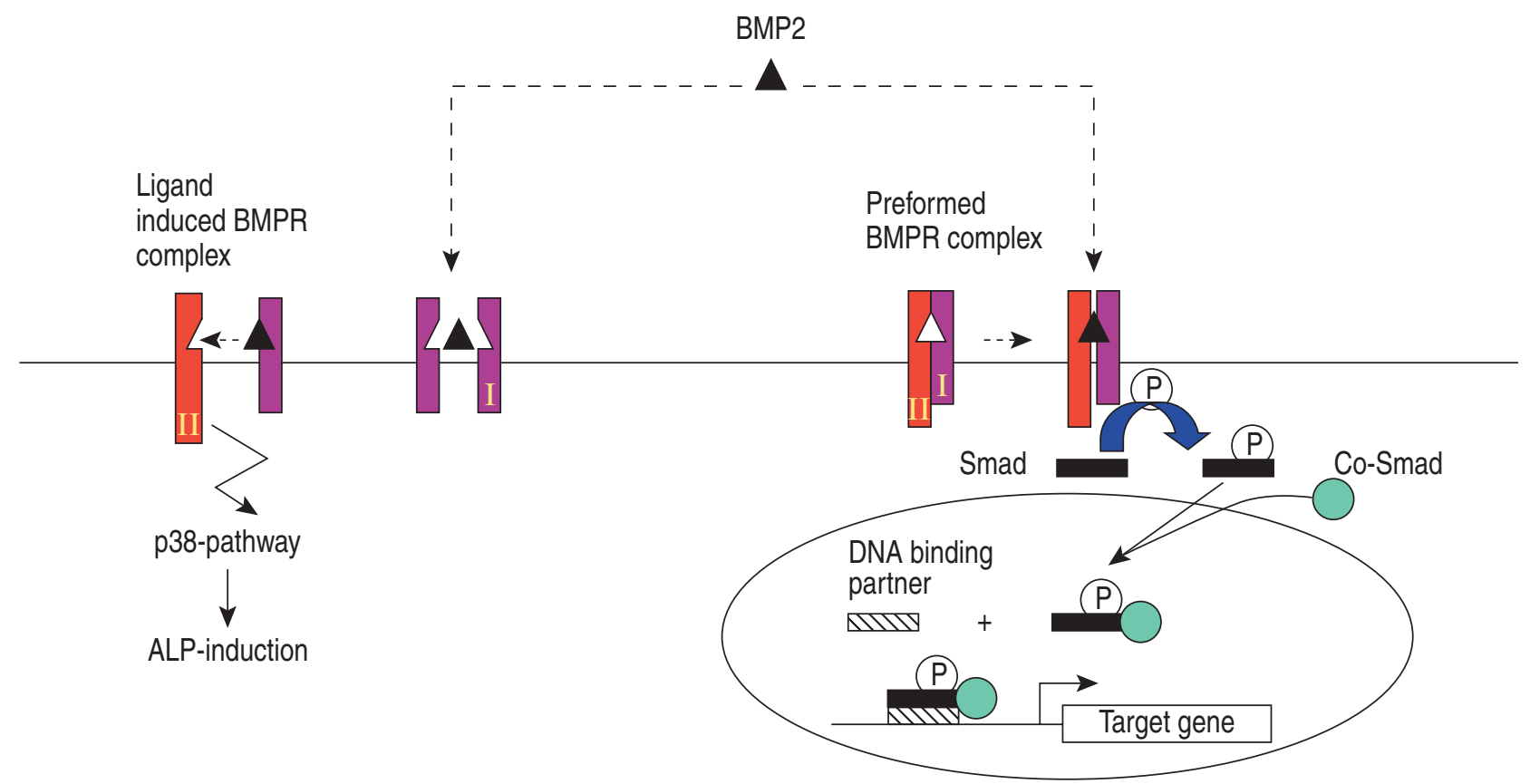

Fig. 1.-Bone morphogenetic protein (BMP) receptor signalling pathways. In the absence of ligand, a significant proportion (30\%) of BMP type I and II receptors (BMPR I and II) exist at the cell surface as heteromeric complexes. Following ligand binding to these preformed complexes, it has been proposed that the type I receptors undergo a ligand-induced conformational change thereby allowing Smad proteins access to the kinase domain of type I receptors. Phosphorylation $(P)$ of Smads leads to their translocation into the nucleus where they activate the transcription of certain genes. A second pathway, independent of Smads, is also activated by the addition of $\mathrm{BMP}^{\prime}$ s through the formation of homomeric type I receptors, which are then able to recruit type II receptors into a heteromeric complex. The activation of this complex leads to alkaline phosphatase (ALP) induction via p38 mitogen activated protein kinase. Co-Smad: coactivator of Smads; DNA: deoxyribonucleic acid.

frameshift mutations have been detected in more than half of the families analysed [16]. These mutations are predicted to interrupt the BMP-mediated signalling pathways, resulting in a predisposition to proliferation rather than apoptosis of cells within small pulmonary arteries. A number of possible explanations exist to account for the PPH families in which BMPR2 gene mutations have not been identified, including mutations in portions of the BMPR2 gene that are not sequenced in these studies (including promoter intronic and 3 '-untranslated regions).

The majority of BMPR2 pathogenic mutations described by MACHADO et al. [16] (59\%) were predicted to lead to a premature truncation of the BMPR-II transcript, most of which will be lost through a process known as nonsense-mediated ribonucleic acid decay. In $36 \%$ of mutations nonconservative amino acid substitutions occurred at highly conserved positions. Using either computer modelling of the structural features of the ligand binding domain or in vitro-expression analysis, these missense mutations were predicted to lead to loss of BMPR-II function. These data strongly support the hypothesis that the predominant molecular mechanism implicated in the dominant inheritance of PPH is one of haploinsufficiency or reduction in the available level of BMPR-II protein [16]. However, in the absence of more detailed analyses an inhibitory effect of the defective proteins upon the normal actions of the BMPR-II complexes, a process referred to as a dominant/negative action, cannot be excluded.
These molecular genetic results suggest that the target cell(s) within the pulmonary arterial wall are sensitive to BMPR2 gene dosage and that the TGF- $\beta$ pathway, mediated through BMPR-II, is critical for the maintenance and/or normal response to injury of the pulmonary vasculature. However, the age of onset of disease both within families and between subjects carrying identical but recurrent mutations is remarkably variable. These findings, together with the previously reported large number of individuals who harbour a deleterious mutation but show no clinical evidence of disease (nonpenetrance), points to the likely requirement for additional factors, either environmental or genetic, in the pathogenesis of the disease. Environmental factors may include drug exposure, as recently suggested in sisters harbouring BMPR2 germline mutations and developing severe PPH after exposure to fenfluramine derivatives [17]. The acquisition of additional genetic factor defects, as in neoplasia, may involve other members of the TGF$\beta$ pathway. Recent microdissection studies of $\mathrm{PPH}$ lesions have suggested that proliferating endothelial cells harbour genetic alterations associated with microsatellite instability and concomitant perturbation of growth and apoptosis gene expression akin to neoplasia [18].

Finally, the marked sex bias for presentation of the disease may implicate either a hormonal component or a role for an X-linked locus in disease predisposition [6]. An alternative suggestion for the observed female predominance was that the genetic defect(s) 
characteristic of the disease might lead to male foetus loss. This was supported by LoyD et al. [8] who showed that more females than males were born to subjects who were obligate carriers for the gene defect, suggesting selective wastage of male foetuses or a distorted primary sex ratio. An abnormal sex ratio of progeny was the first clinical finding which pointed to the possibility that the PPH gene may have a role in embryonic development. A significant trend for the disease to develop at a younger age in members of subsequent generations has also been reported [8]. This manifestation, known as genetic anticipation, is reminiscent of the phenomena associated with increasing instability of trinucleotide repeat amplification, which forms the basis for several neurological diseases, including fragile $\mathrm{X}$ syndrome, myotonic dystrophy, and Huntington's disease. Anticipation in familial PPH was confirmed by LoYD et al. [8], showing that the age $( \pm \mathrm{SD})$ at death was $45.6 \pm 14.5$ versus $36.3 \pm 12.6$, and $24.2+11$ yrs in successive generations. To date no molecular genetic evidence to explain anticipation in PPH has been presented. It remains possible that an alternative explanation for this phenomenon in PPH, might reflect increased environmental exposure, rather than a genetic mechanism.

Arterial remodelling in $\mathrm{PPH}$ involves hypertrophy and proliferation of endothelial, smooth muscle cells, as well as fibroblasts, but how defects in BMPR-II contribute to these changes is currently unknown. However, BMP2 has been shown to inhibit growth of cultured aortic vascular smooth muscle cells indicating they have an intact BMP signalling pathway and that defects in BMP-mediated signalling may predispose to proliferation under certain environmental conditions. Recently, Morrell et al. [19] have demonstrated functional abnormalities of pulmonary artery smooth muscle cells isolated from $\mathrm{PPH}$ patients, including resistance to the antiproliferative effects of TGF- $\beta 1$ and BMPs. The TGF- $\beta$ superfamily plays a critical role in the development and maintenance of the integrity of the normal vasculature. Thus, the altered cellular responses may contribute to the formation of lesions that lead to the vascular obliteration observed in the pulmonary arteries of patients with PPH.

\section{Sporadic primary pulmonary hypertension}

In both familial and sporadic PPH patients, histological examination of pulmonary arteries shows increased muscularisation of pulmonary arteries, intimal hyperplasia, endothelial cell proliferation, and in situ thrombosis. These findings, together with the similar clinical features and progression of the disease and the determination of previously unknown co-ancestry between so-called sporadic PPH patients, suggest that a proportion of so-called sporadic PPH patients may indeed have the same underlying molecular genetic defect as seen in familial PPH. This is further supported by the fact that the disease gene, although inherited as an autosomal dominant trait, acts with low penetrance, hence in the absence of detailed genealogical data, familial cases may be easily overlooked.

Two recent studies have clearly demonstrated that so-called sporadic PPH may indeed represent unidentified familial cases. First, a search on several American families has uncovered a kindred affected by familial PPH that spans seven generations and involves five subfamilies initially not known to be related [20]. This kindred included 12 affected members who were initially thought to have sporadic PPH and seven affected members whose conditions were first misdiagnosed as other cardiopulmonary diseases. Mutational analysis of the BMPR2 gene in six affected members revealed that all had the same missense mutation in exon 6. Further genealogical investigations revealed that all these families were in fact related, with common ancestors born in the nineteenth century. The discovery of this very large kindred affected by familial PPH supports the hypothesis that cases of presumed sporadic PPH are in fact inherited [20]. As resolution of this question has important implications for patient diagnosis, genetic counselling and clinical screening, the BMPR2 gene has been investigated in a cohort of 50 unrelated patients with a clinical diagnosis of $\mathrm{PPH}$ and no identifiable family history of PPH, using direct sequencing of the entire coding region and intron/ exon boundaries of the BMPR 2 gene [21]. In addition, deoxyribonucleic acid (DNA) from available parent pairs was used to assess the occurrence of spontaneous (de novo) mutations contributing to sporadic PPH. A total of 11 different heterozygous germline mutations of the BMPR2 gene were identified in 13 of the $50 \mathrm{PPH}$ patients studied, including missense $(\mathrm{n}=3)$ nonsense $(n=3)$, and frameshift $(n=5)$ mutations each predicted to alter the cell signalling response to specific ligands and hence of functional significance. Parental analysis revealed three occurrences of paternal transmission and two of de novo (spontaneous) mutation of the BMPR2 gene in sporadic PPH (fig. 2). Therefore, the sporadic form of $\mathrm{PPH}$ is associated with germline mutations of the gene encoding the receptor protein BMPR-II in $\geqslant 26 \%$ of cases [21]. This suggests that molecular description of $\mathrm{PPH}$ patients, based upon the presence or absence of BMPR2 mutations may have important implications for screening of relatives.

\section{Acquired genetic defects in PPH}

LEE et al. [22] have shown that endothelial cells within PPH plexiform lesions demonstrate monoclonality, whereas PAH lesions develop via polyclonal expansion of endothelial cells in scleroderma or congenital systemic to pulmonary shunts. Microdissection studies by YEAGER et al. [18] recently demonstrated that endothelial cells in PPH lesions are genetically unstable with $50 \%$ of lesions demonstrating microsatellite instability. Endothelial cells in PPH lesions harbour mutations permissive for clonal cell growth, including mutation of at least one allele of TGF- $\beta$ receptor type II in one third of the lesions from sporadic PPH individuals. These results suggest 
a)

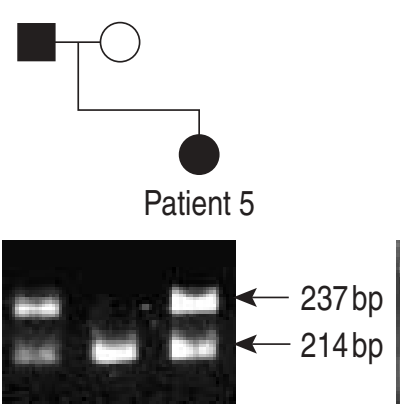

b)

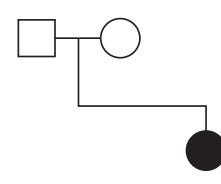

Patient 10

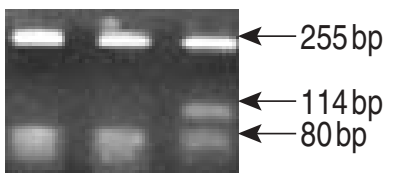

Fig. 2.-Assessment of mutation origin in patients with sporadic primary pulmonary hypertension (PPH). a) Detection of sequence variant segregation in the parents of sporadic patient 5 by restriction endonuclease digestion with $\operatorname{Taq} \mathrm{I}$, as the mutation creates a restriction site, followed by restriction fragment analysis on a $3 \%$ agarose gel. The mutation is present in the unaffected paternal sample. Observed fragment sizes are indicated beside the gel image. Squares indicate males, circles indicate females. Filled symbols indicate patients carrying germline bone morphogenetic protein receptor type II gene (BMPR2) mutations either unaffected by PPH (father) or displaying PPH (daughter). Open symbols indicate individuals displaying neither $\mathrm{PPH}$ nor germline BMPR2 mutations. b) Detection of mutation segregation in the parents of sporadic patient 10 by restriction fragment analysis on a $3 \%$ gel, following digestion with the enzyme Fnu 4HI. The mutation creates a restriction site. In this instance, the mutation is de novo. Observed fragment sizes are indicated beside the gel image. Squares indicate males, and circles indicate females. Filled symbols indicate patients carrying germline BMPR2 mutations. Open symbols indicate individuals displaying neither PPH not germline BMPR2 mutations. In both a) and b) deoxyribonucleic acid (DNA) bands corresponding to the paternal and maternal samples, as well as the PPH cases are displayed directly below the corresponding symbol on the genetic tree.

that in some sporadic PPH patients proliferating endothelial cells have acquired (somatic) genetic alterations.

\section{Other forms of pulmonary arterial hypertension}

Exposure to appetite suppressants aminorex, fenfluramine and dexfenfluramine is a definite risk factor for PAH $[2,4,5]$. Increased risk for PAH was first reported in the late sixties with exposure to aminorex and in the mid-nineties with fenfluramine or dexfenfluramine. However, the exact mechanism(s) by which fenfluramine derivatives promote $\mathrm{PAH}$ has not been established [23]. Individual susceptibility factors are likely to play an important role since the absolute risk in the general population is low (around 1 case for 10,000 individuals exposed to fenfluramine derivatives). BMPR 2 mutations were determined in patients with sporadic PAH who had taken fenfluramine derivative [17]. BMPR2 mutations that predict changes in the primary structure of the BMPR-II protein were found in $\geqslant 9 \%$ of $\mathrm{PAH}$ patients with a history of fenfluramine derivatives intake [17]. This differed significantly from normal healthy French controls, in whom no BMPR2 mutations could be identified. Moreover, the patients with mutations of the BMPR2 gene had a significantly shorter duration of exposure to fenfluramines as compared to patients with no evidence of mutations [17]. Therefore, a proportion of patients with fenfluramine derivativeassociated PAH have BMPR2 mutations. These BMPR2 defects do not rule out a role for additional mechanisms triggering the occurrence of the disease, including drug-induced alteration of the serotonin pathway. The possible relevance of serotonin in fenfluramine-induced PAH is supported by the fact that fenfluramine derivatives induce valvular heart disease very similar to carcinoid syndrome and that a decrease in platelet serotonin storage with enhanced blood concentration of free serotonin has been reported in sporadic cases of PPH [23]. Further investigations should determine whether abnormal TGF- $\beta$ signalling alone, or in combination with other factors, plays a role in the occurrence of $\mathrm{PAH}$ associated with fenfluramine derivative intake.

As discussed above, the current classification of PAH recognises the clinical, pathophysiological and therapeutical similarities between $\mathrm{PPH}$ and $\mathrm{PAH}$ associated with, for example, collagen vascular diseases, HIV infection, portal hypertension, congenital systemic to pulmonary shunts, and anorexigen exposure. The present authors and others have systematically assessed patients with well-characterised conditions associated with PAH. The only patient population in which BMPR2 mutations could be identified was that of fenfluramine-exposed individuals. In conclusion, despite striking similarities between PAH variants, germline BMPR2 genetic defects have only been identified to date in inherited cases of PPH and fenfluramine-associated cases.

\section{Hereditary haemorrhagic telangiectasia (Osler-Weber-Rendu syndrome)}

Other components of the TGF- $\beta$ receptor pathway have been implicated in hereditary haemorrhagic telangiectasia. Hereditary haemorrhagic telangiectasia (Osler-Weber-Rendu syndrome) is an autosomal dominant vascular dysplasia characterised by mucocutaneous telangiectases affecting the nasal and gastrointestinal mucosa, lips, tongue, fingertips, and macroscopic arteriovenous malformations [24]. These lead to recurrent epistaxis and gastrointestinal blood loss, as well as arteriovenous malformations, particularly in the pulmonary, hepatic, and cerebral circulation. Pulmonary artriovenous malformations create clinically significant right-to-left shunts, causing hypoxaemia, orthodeoxia, platypnoea, chest murmurs, paradoxical embolism, stroke, and cerebral abscesses. In this setting the occurrence of pulmonary hypertension is usually due to the hyperkinetic state promoting "high cardiac output heart failure" (venous "postcapillary" pulmonary hypertension) [24]. However, PAH that is clinically and histologically undistinguishable from PPH may occur in this patient population [25].

Mutations in two genes encoding family members of the TGF- $\beta$ receptor pathway underlie hereditary haemorrhagic telangiectasia $[15,24]$. These genes code for a type I receptor (activin-receptor-like 
kinase 1 (ALK-1), located on chromosome 12) and a type III receptor (accessory) (endoglin, located on chromosome 9), respectively. Clinical analysis of five kindreds plus one individual patient with hereditary haemorrhagic telangiectasia led to the identification of $10 \mathrm{PAH}$ cases [25]. These subjects suffered with "precapillary" pulmonary hypertension confirmed by right-heart catheterisation demonstrating elevated mean pulmonary artery pressure, low pulmonary artery wedge ("capillary") pressure, low cardiac output and markedly elevated pulmonary vascular resistances. Family members spanning the whole spectrum of both conditions could be studied (i.e. unaffected family members, and family members with $\mathrm{PAH}$, hereditary haemorrhagic telangiectasia, or both) (fig. 3). Analysis of genes encoding TGF- $\beta$ receptor proteins, including ALK-1, endoglin, and BMPR2, allowed the demonstration that $\mathrm{PAH}$ in association with hereditary haemorrhagic telangiectasia can involve mutations in ALK1 [25] (fig. 3). Lastly, immunohistochemical analysis of paraffin-embedded lung sections from a subject with plexiform PAH and mutations in ALK1 demonstrated the presence of ALK-1 protein product in diseased pulmonary vascular endothelium [25]. This later finding lends support for the suggestion that endothelium cell dysfunction is crucial to the pathogenesis of both hereditary haemorrhagic telangiectasia and PAH. TGF- $\beta$ signalling seems to affect both vascular differentiation and proliferation. The pleiotropic nature of TGF- $\beta$ as a growth factor offers a potential explanation for the variable complications of hereditary haemorrhagic telangiectasia. The net effect of ALK1 dysfunction may depend on local vascular interactions and other environmental or genetic factors.

\section{Family A}

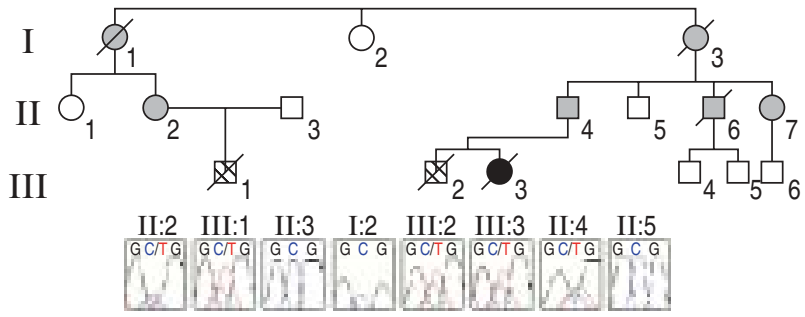

Fig. 3.-Segregation of missense mutation in the activin-receptorlike kinase (ALK1) gene in a family with hereditary haemorrhagic telangiectasia and pulmonary hypertension. Segregation of missense mutation in the ALK1 gene, generated by a cytosine to thymine transition at nucleotide position 1450 (exon 10), in family A. Cosegregation of the disease with hereditary haemorrhagic telangiectasia and pulmonary hypertension in the family was resolved by direct sequencing of individuals for whom deoxyribonucleic acid was available. The black filled symbols indicate individuals with both pulmonary hypertension and hereditary haemorrhagic telangiectasia, grey filled symbols are individuals with only hereditary haemorrhagic telangiectasia, striped symbols refer to individuals with only pulmonary hypertension and open symbols are individuals unaffected by either condition. Squares indicate males, and circles indicate females. Diagonal lines through symbols indicate deceased individuals. G: guanine; C: cytosine; T: thymine.

\section{The serotonin hypothesis}

"Unexplained" pulmonary hypertension in patients with rare diseases provides valuable tools to analyse a possible relevant pathobiological basis of PAH. Development of severe PAH with no identifiable cause in a patient with a rare familial platelet storage deficiency allowed HeRvé et al. [26] to hypothesise that serotonin, a pulmonary vasoconstrictor and growth factor for vascular smooth muscle cells stored in platelets, could play a critical role in PAH. This observation subsequently led to the description of altered serotonin turnover leading to an increased availability of free serotonin in patients with $\mathrm{PPH}$ [27].

Recent studies have extended the hypothesis that serotonin may play a critical role in PPH. Hyperplasia of pulmonary artery smooth muscle cells is a pathological hallmark of PPH. EDDAHIBI et al. [28] recently showed that pulmonary artery smooth muscle cells from PPH patients grow faster than pulmonary artery smooth muscle cells from controls when stimulated by serotonin or serum. These effects were due to an increased expression of the serotonin transporter (5HTT) which mediates internalisation of indoleamine [28]. In the presence of 5HTT inhibitors the growth stimulatory effects of serum and serotonin were markedly reduced and a difference between the growth of pulmonary artery smooth muscle cells from patients and controls was no longer observed. As compared with controls the expression of 5HTT was increased in cultured pulmonary artery smooth muscle cells as well as in platelets and lungs from patients with PPH, where it predominated in the media of thickened pulmonary arteries and in onion-bulb lesions. The L-allelic variant of the gene promoter, which is associated with 5HTT overexpression and increased pulmonary artery smooth muscle cell growth was present in $65 \%$ of $\mathrm{PPH}$ patients but in only $27 \%$ of controls [28]. Therefore, 5HTT may play a significant role in the pathogenesis of pulmonary artery smooth muscle cells proliferation in PPH, and a 5HTT polymorphism could confer susceptibility to $\mathrm{PPH}$.

\section{Type Ia glycogen storage disease}

The present authors have recently described in detail patients who developed PAH in the context of other rare diseases, allowing the analysis of novel pathophysiological pathways to be extended. For instance, hereditary haemorrhagic telangiectasia supported and extended the concept of altered TGF- $\beta$ signalling in the context of severe precapillary pulmonary hypertension [25]. More recently, patients displaying severe PAH and type I glycogen storage disease allowed further investigation of the possible promoting role of metabolic disturbances in $\mathrm{PAH}$ patients [29].

Type Ia glycogen storage disease is an autosomal recessive disorder caused by a deficiency of glucose6-phosphatase (type Ia, von Gierke's disease) or glucose-6-phosphate translocase (type Ib). Its estimated incidence is 1 per 100,000 . Since the pioneering 
description of PIzzo [30] in 1980, seven cases of severe pulmonary hypertension have been described in patients with type I glycogen storage disease [29, 30]. In all but one case this condition was extremely severe with a fatal outcome within months of cardiopulmonary arrest and/or untractable congestive right heart failure. When available, post-mortem examination of the lungs demonstrated pulmonary hypertensive arteriopathy, as previously described in $\mathrm{PPH}$. The only case occurring before the age of 10 was in a young girl with atrial septal defect of the secundum type [31]. Interestingly, neither atrial septal defect nor type Ia glycogen storage disease typically lead to severe pulmonary hypertension in the first decade, suggesting that these conditions could have cumulative negative effects with a faster progression of the pulmonary vascular disease. The six remaining cases of severe PAH complicating the course of type I glycogen storage disease occurred in the second or third decade, suggesting that long-term metabolic abnormalities may contribute to the long latency of pulmonary vascular disease [29]. In his initial report PIzzo [30] suggested that the occurrence of pulmonary arterial hypertension in type I glycogen storage disease could be due to an abnormal production of vasoconstrictive amines such as serotonin. The present authors recently investigated a case of type I glycogen storage disease with severe PAH in the absence of BMPR2 germline mutations, and a series of 13 patients with type I glycogen storage disease and no evidence of pulmonary vascular disease [29]. Elevated plasma serotonin concentrations were found in patients with severe PAH and/or type I glycogen storage disease, as compared with controls. The present authors hypothesised that type I glycogen storage disease could represent another condition where abnormal handling of serotonin is one event in a multistep process leading to PAH. Interestingly, no 5HTT polymorphism putatively conferring susceptibility to PPH could be identified in patients with elevated plasma serotonin concentrations and type I glycogen storage disease (J-M Launay, Dept of Biochemistry, Hôpital Lariboisière, Assistance Publique, Hôpitaux de Paris, France, personal communication).

\section{The future}

\section{Genetic counselling}

Because the risk for disease in first-degree relatives of a newly diagnosed patient with PPH is low, uncertainty has persisted regarding appropriate strategies for clinical screening of at-risk family members [2]. It will be of considerable importance to ensure that the use of genetic analysis in the diagnosis of PPH be supported by appropriate genetic counselling, as part of an integrated program of family-based screening. As BMPR2 mutations are multiple and distinct between different families, geneticists need first to analyse the BMPR2 gene from the affected patient. This may be possible even if the affected proband is deceased by using a stored blood or tissue sample. As indicated above BMPR2 mutations have been found in $\sim 60 \%$ of familial cases and $30 \%$ of so-called sporadic cases [16, 21]. If no mutation is identified the information given to the relatives differs between sporadic and familial PPH. In case of sporadic PPH one should assume that the inherited risk is low, marginally increased from that of the general population. In the case of familial PPH one may use the family tree to identify an obligatory carrier of an as-yet unidentified mutation. For other relatives the risk cannot be easily defined and advice is presently dependent upon local practice. If a BMPR2 mutation is detected, it will be possible to test relatives to assess whether they have inherited the mutation or not. In relatives where the identified mutation is not detected, the risk of developing $\mathrm{PPH}$ is very low, not different than that of the general population. Conversely, the risk of developing PPH amongst relatives harbouring a BMPR2 mutation is significantly increased compared to background rates, but remains low, as the disease alleles act with reduced penetrance. Present estimates suggest $\leqslant 20 \%$ life-time risk of developing PPH for BMPR 2 mutation carriers. Further work is required to clarify this.

Prior to any predictive test, it is essential that consultants meet with a genetic counsellor to consider the possible implications of a positive test result, including life, health insurance, employment and psychological aspects. With particular relevance to $\mathrm{PPH}$, it will also be important to discuss the clinical tests that may be undertaken to look for evidence of the disease in case of a positive genetic test. These may include echocardiography, stress echocardiography, right-heart catherterisation at rest or at exercise. With regards to testing of children, the present authors would presently recommend that each situation should be considered in detail with all parties involved in the care of such families. More data are urgently needed to generate a clear view on this important issue before providing guidelines. Lastly, it will be critical to obtain an expert agreement on recommendations regarding risk factors of $\mathrm{PAH}$ in individuals harbouring BMPR2 mutations. This is will be particularly difficult for situations known to induce additional risk of $\mathrm{PAH}$, including pregnancy. Indeed pregnancy combines the particular risk of destabilising a latent pulmonary vascular condition, as well as transmission of the gene to the offspring. Preimplantation genetic diagnosis whilst not yet reported is now technically possible in families with identified mutations.

\section{Pharmacogenetics}

Considerable interindividual variation exists at the DNA level in targets for medication, and variability in (positive or negative) response to treatment may, at least in part, be determined by this genetic variation [32]. Genetic variability at the DNA level occurs in $\sim 1$ in 500 to 1 in 1,000 bases of coding DNA and in 1 in 300 to 1 in 500 bases in noncoding DNA. 
The function of any given polymorphism depends on its nature and position. Polymorphisms at the DNA level can be either synonymous or nonsynonymous, the latter implying that the polymorphism produces an amino-acid substitution in the relevant protein product.

Higenbottam et al. [33] have recently tested the hypothesis that there is an over representation of the cytochrome P450 CYP2D6 genotype amongst patients who developed fenfluramine-associated PAH. Indeed, fenfluramine derivatives are metabolised to a significant extent by CYP2D6, an enzyme that shows a marked genetic polymorphism such that $8 \%$ of White people ("poor metaboliser") lack a functional form. Inability to eliminate fenfluramines efficiently might be associated with a greater risk of developing PAH after drug exposure. The frequency of the poor metaboliser genotype was indeed significantly greater in PAH patients with a history of fenfluramine exposure $(20 \%)$ as compared to the sporadic PPH group (5\%), and normal healthy controls (7\%) [33]. Therefore, the poor metaboliser genotype for CYP2D6 could be associated with an increased risk of developing $\mathrm{PAH}$ from treatment with fenfluramine and dexfenfluramine.

Similarly, it is well established that only a minority of PAH patients will have an acute and/or chronic vasodilator response to inhaled nitric oxide, intravenous prostacyclin or oral calcium channel blockers [34]. There is unfortunately no clinical, demographical or baseline haemodynamic characteristic predictive of a vasodilator response to treatment. Whether novel pharmacogenetic tools will provide useful information allowing noninvasive identification of patients with a possible vasodilator response remains to be demonstrated. In asthma, many clinical studies have been performed that examine the potential effects of known polymorphisms and in general they have shown relatively small effects, although there are reasonably convincing data supporting reduced bronchodilator responses in individuals carrying some polymorphic variants of the $\beta 2$-adrenoreceptor. Similar studies analysing polymorphic variants of known drug targets including prostaglandins and endothelins should now be performed in PPH.

\section{Molecular classification}

The identification of germline mutations of the BMPR2 gene as the inherited basis of PPH has important implications for assessment and management of patients with this dramatic vascular disease. However, the detailed molecular mechanisms associated with the pathogenesis of the disorder will require further analysis, including identification of target genes whose transcription is regulated by BMPR-II-mediated cell signalling. The demonstration that gene dosage and receptor activity are critical will aid the development of functional experiments designed to delineate the detailed molecular pathology of PPH.

\section{Conclusion}

The last couple of years have witnessed an outstanding number of major genetic discoveries allowing a better understanding of the pathophysiology of this devastating orphan disease. Much of this novel information emphasises the relevance of the transforming growth factor- $\beta$ signalling pathway in pulmonary arterial hypertension [15]. While this achievement has generated extreme interest, the pathobiology of severe pulmonary arterial hypertension remains unclear and genomic approaches to pulmonary hypertension research may identify additional contributory molecular pathways, implicating, for example, vasoactive agents, cytokines, chemokines and growth factors [3]. Future research strategies should aim primarily at identifying novel therapeutic targets aimed at improving clinical care in this condition. Lastly, there is an urgent need to develop validated guidelines for genetic counselling to help families and pulmonary vascular specialists to cope with this difficult disorder.

\footnotetext{
Acknowledgements. The authors wish to express their thanks to the many patients and their parents who provided samples and details of their medical and family histories. R.C Trembath thanks R. Machado, J. Morton and R. Harrison for work described in this review.
}

\section{References}

1. Rubin LJ. Primary pulmonary hypertension. $N$ Engl J Med 1997; 336: 111-117.

2. Rich S. Primary pulmonary hypertension: executive summary from the World Symposium, Primary pulmonary hypertension 1998. Available from the World Health Organization via the Internet. www.who.int/ncd/cvd/pph.htlm. Date last updated: July 3, 2001. Date last accessed: July 2001.

3. Archer S, Rich S. Primary pulmonary hypertension: a vascular biology and translational research "Work in progress". Circulation 2000; 102: 2781-2791.

4. Fishman AP. Clinical classification of pulmonary hypertension. Clin Chest Med 2001; 22: 385-391.

5. Abenhaim L, Moride Y, Brenot F, et al. Appetitesuppressant drugs and the risk of primary pulmonary hypertension. N Engl J Med 1996; 335: 609-616.

6. Humbert M, Nunes H, Sitbon O, Parent F, Hervé P, Simonneau G. Risk factors for pulmonary arterial hypertension. Clin Chest Med 2001; 22: 459-475.

7. Dresdale DT, Mitchom RJ, Schultz M. Recent studies in primary pulmonary hypertension, including pharmacodynamic observations on pulmonary vascular resistance. Bull NY Acad Med 1954; 30: 195-207.

8. Loyd JE, Butler MG, Foroud TM, Conneally PM, Phillips JA 3rd, Newman JH. Genetic anticipation and abnormal gender ratio at birth in familial primary pulmonary hypertension. Am J Respir Crit Care Med 1995; 152: 93-97.

9. Rich S, Dantzker DR, Ayres SM, et al. Primary pulmonary hypertension: a national prospective study. Ann Int Med 1987; 107: 216-223. 
10. Nichols WC, Koller DL, Slovis B, et al. Localization of the gene for familial primary pulmonary hypertension to chromosome 2q31-32. Nat Genet 1997; 15: 277-280.

11. Lane KB, Machado RD, Pauciulo MW, et al. Heterozygous germline mutations in a TGF- $\beta$ receptor, BMPR2, are the cause of familial primary pulmonary hypertension. The International $\mathrm{PPH}$ Consortium. Nat Genet 2000; 26: 81-84.

12. Deng Z, Morse JH, Slager SL, et al. Familial primary pulmonary hypertension (gene PPH1) is caused by mutations in the bone morphogenetic protein receptor-II gene. Am J Hum Genet 2000; 67: 737-744.

13. Massague J, Chen YG. Controlling TGF- $\beta$ signaling. Genes Dev 2000; 14: 627-644.

14. Wilkins MR, Gibbs JSR, Shovlin CL. A gene for primary pulmonary hypertension. Lancet 2000; 356: 1207-1208.

15. Loscalzo J. Genetic clues to the cause of primary pulmonary hypertension. N Engl J Med 2001; 345: 367-371.

16. Machado RD, Pauciulo MW, Thomson JR, et al. BMPR2 haploinsufficiency as the inherited molecular mechanism for primary pulmonary hypertension. Am J Hum Genet 2001; 68: 92-102.

17. Humbert M, Deng Z, Simonneau G, et al. BMPR2 germline mutations in pulmonary hypertension associated with fenfluramine derivatives. Eur Respir $J$ 2002; 20: 518-523.

18. Yeager ME, Halley GR, Golpon HA, Voelkel NF, Tuder RM. Microsatellite instability of endothelial cell growth and apoptosis genes within plexiform lesions in primary pulmonary hypertension. Circ Res 2001; 88: e2-e11.

19. Morrell NW, Yang X, Upton PD, et al. Altered growth responses of pulmonary artery smooth muscle cells from patients with primary pulmonary hypertension to transforming growth factor- $\beta 1$ and bone morphogenetic proteins. Circulation 2001; 104: 790795.

20. Newman JH, Wheeler L, Lane KB, et al. Mutation in the gene for bone morphogenetic protein receptor II as a cause of primary pulmonary hypertension in a large kindred. N Engl J Med 2001; 345: 319-324.

21. Thomson JR, Machado RD, Pauciulo MW, et al. Sporadic primary pulmonary hypertension is associated with germline mutations of the gene encoding BMPR-II, a receptor member of the TGF- $\beta$ family. J Med Genet 2000; 37: 741-745.

22. Lee SD, Shroyer KR, Markham NE, Cool CD,
Voelkel NF, Tuder RM. Monoclonal endothelial cell proliferation is present in primary but not secondary pulmonary hypertension. J Clin Invest 1998; 101: 927934.

23. Simonneau G, Fartoukh M, Sitbon O, Humbert M, Jagot J-L, Hervé P. Primary pulmonary hypertension associated with the use of fenfluramine derivatives. Chest 1998; 114: 195S-198S.

24. Shovlin CL, Letarte M. Hereditary hemorrhagic telangiectasia and pulmonary arteriovenous malformations: issues in clinical management and review of pathogenic mechanisms. Thorax 1999; 54: 714-729.

25. Trembath $\mathrm{R}$, Thomson JR, Machado RD, et al. Clinical and molecular genetic features of pulmonary hypertension in patients with hereditary hemorrhagic telangiectasia. N Engl J Med 2001; 345: 325-334.

26. Hervé $\mathrm{P}$, Drouet $\mathrm{L}$, Dosquet $\mathrm{C}$, et al. Primary pulmonary hypertension in a patient with a familial platelet storage pool. Am J Med 1990; 89: 117-120.

27. Hervé P, Launay JM, Scrobohaci ML, et al. Increased plasma serotonin in primary pulmonary hypertension. Am J Med 1995; 99: 249-254.

28. Eddahibi S, Humbert M, Fadel E, et al. Serotonin transporter overexpression is responsible for pulmonary artery smooth muscle hyperplasia in primary pulmonary hypertension. J Clin Invest 2001; 108: $1141-1150$.

29. Humbert M, Labrune P, Sitbon O, et al. Pulmonary arterial hypertension and type I glycogen storage disease: the serotonin hypothesis. Eur Respir J 2002; 20: 59-65.

30. Pizzo CJ. Type I glycogen storage disease with focal nodular hyperplasia of the liver and vasoconstrictive pulmonary hypertension. Pediatrics 1980; 65: 341-343.

31. Bolz D, Stocker F, Zimmermann A. Pulmonary vascular disease in a child with atrial septal defect of the secundum type and type I glycogen storage disease. Pediatr Cardiol 1996; 17: 265-267.

32. Hall IP. Pharmacogenetics, pharmacogenomics and airway disease. Respir Res 2002; 3: 10.

33. Higenbottam T, Humbert M, Simonneau G, et al. Subjects deficient for CYP2D6 expression (poor metabolisers) are over-represented among patients with anorectic associated pulmonary hypertension (abstract). Am J Respir Crit Care Med 1999; 159: A165.

34. Sitbon O, Humbert M, Jagot J-L, et al. Inhaled nitric oxide as a screening agent to safely identify responders to oral calcium-channel blockers in primary pulmonary hypertension. Eur Respir J 1998; 12: 265-270. 